\title{
Absolute Proton Polarimetry at RHIC
}

\section{Oleg Eyser ${ }^{1}$ for the RHIC/CNI polarimetry group}

Brookhaven National Laboratory

Upton, NY 11973, USA

E-mail: keyserabnl.gov

The Relativistic Heavy Ion Collider (RHIC) has successfully collided polarized proton beams with momenta as high as $255 \mathrm{GeV} / \mathrm{c}$ and average polarizations up to $\mathrm{P}=60 \%$. The polarization of the proton beams is vital input to any of the spin-dependent measurements at the RHIC experiments. The absolute beam polarization is determined through spin dependent elastic scattering with a polarized hydrogen jet target. Recent improvements of beam luminosities and a new set of silicon detectors have enabled studies of systematic effects in the polarimeters with unprecedented accuracy. This paper presents the latest polarimetry results with protons beams of 100 and $255 \mathrm{GeV} / \mathrm{c}$. This includes a discussion of the polarimeter performance in the presence of heavy ion beams for the first time during the recently concluded RHIC run 2015.

XVIth International Workshop in Polarized Sources, Targets, and Polarimetry, PSTP2015,

14-18 September 2015,

Bochum Germany

\section{${ }^{1}$ Speaker}

(C) Copyright owned by the author(s) under the terms of the Creative Commons

Attribution-NonCommercial-NoDerivatives 4.0 International License (CC BY-NC-ND 4.0). 


\section{Introduction}

The Relativistic Heavy Ion Collider, RHIC, at Brookhaven National Laboratory provides collisions of polarized protons at center of mass energies up to $500 \mathrm{GeV}$. It is this unique capability that requires careful relative monitoring of the beam polarization throughout the storage times and an absolute normalization in particular at the previously unexplored highest energies. Typical RHIC fills are using about 110 filled bunches of $1.5 \cdot 10^{11}$ protons with alternating vertical polarization directions of adjacent bunches (or pairs of bunches). The absolute polarization is measured non invasively with a polarized atomic hydrogen target, HJET, throughout the whole store. In addition, the polarization life time is tracked at several points during the store with Carbon fiber targets, typically every two to three hours (see [1] for a detailed discussion of the Carbon polarimetry). These proceedings show first results from the 2015 RHIC run. This includes mainly data from proton-proton operation at $\sqrt{s}=200 \mathrm{GeV}$, but also some additional studies from the consecutive proton-ion run.

\section{Elastic Proton Scattering: Detector Setup and Calibrations}

The polarimetry at RHIC is generally based on elastic scattering of beam protons on targets of either atomic hydrogen or Carbon nuclei. The recoil particle is detected in Silicon strips which are located transverse to the beam direction, see Figure 1. The detectors measure the time of flight as well as the energy of the particle in a kinematic region where the elastic signal is easy to select. The HJET is using a combination of eight detectors to measure recoil protons from both beams. Each detector consists of 12 strips with $3.75 \mathrm{~mm}$ pitch, covering an area of $4.5 \times 5.0 \mathrm{~cm}^{2}$ downstream of the jet target. The strips have a thickness of $500 \mu \mathrm{m}$ and are able to stop protons with an energy of less than about 7.0 MeV. Higher energies will start to

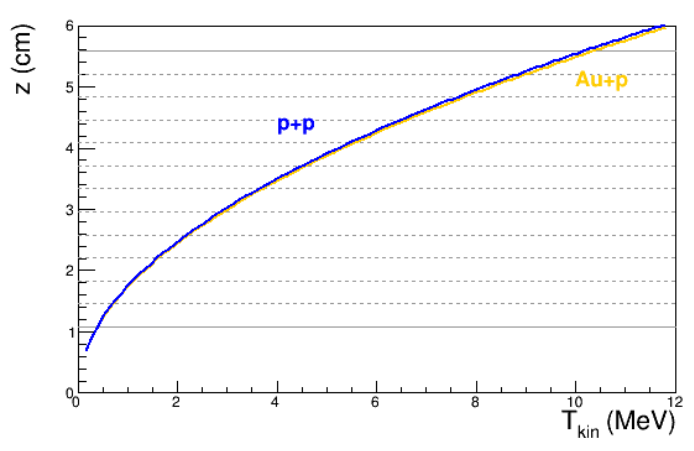

Figure 1: Deflection of the recoil proton from elastic proton scattering with a $100 \mathrm{GeV}$ incident beam on the HJET target. The horizontal lines represent the acceptance of the Silicon strip detectors (situated 75 $\mathrm{cm}$ transverse to the beam direction). punch through the detector and only leave a fraction of their energy, but this only affects the two strips that are situated most downstream from the target. For the current work, we do not consider the punch through protons and remove them from the analysis. The detectors are read out with FADC cards which store the full waveform with a $2.4 \mathrm{~ns}$ resolution (see [2] for more details of the upgraded setup and the treatment of punch through particles). Energy and time of flight are later determined from the waveform in the offline analysis.

For the energy calibration, two radioactive $\alpha$-sources of known energy are used (Gd-148, $\mathrm{E}_{\alpha}=3.183 \mathrm{MeV}$ and Am-241, $\mathrm{E}_{\alpha}=5.486 \mathrm{MeV}$ ). Calibrations were typically carried out every few days to ensure gain stability and track possible changes. The proper time of flight is adjusted for possible drifts of the delay times in cables and readout electronics over the many months of operation. 
The elastic signal is selected for the known kinematics of the recoil proton. At RHIC energies, the recoil angle is mainly dependent on the beam momentum. This proved to be especially advantageous during $\mathrm{p}+\mathrm{Au}$ and $\mathrm{p}+\mathrm{Al}$ operations, when the elastic recoil from the heavy ion beam could be used for additional monitoring of the target performance and possible effects of background on the determination of the proton beam polarization. The kinematic

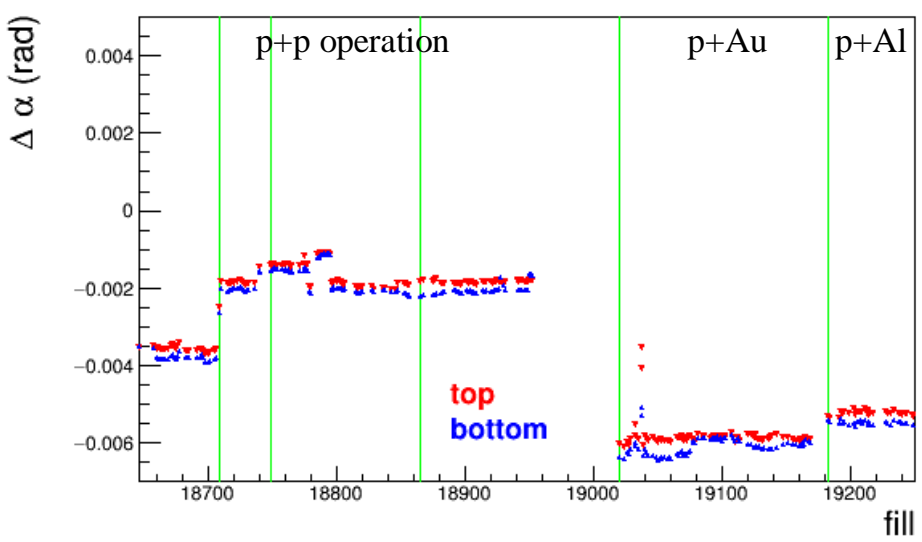

Figure 2: Difference of the actual detector position and the reconstructed position from the missing mass of the elastically scattered proton per RHIC fill. Plotted are two (top and bottom) out of the total of eight detectors. Vertical lines are documented adjustments to the magnetic holding field and major changes of the accelerator parameters. selection is very sensitive to the detector alignment, also. While this includes an unlikely motion of the detectors themselves, the main contributions here are in fact changes in the accelerator parameters to tune the stability of the beam and the beam polarization. In particular during the heavy ion operation, the rigidity of the accelerator led to a significant difference of the beam angle on the jet target. These changes on the acceptance of left and right side detectors can be corrected with adjustments to the magnetic holding field of the target. The offline analysis determines the alignment of the detectors for each RHIC fill and applies a correction for the elastic signal selection, see Figure 2.

\section{Asymmetries and Background}

At nonrelativistic energies, elastic proton scattering exhibits a spin dependent transverse asymmetry or analyzing power, $\mathrm{A}_{\mathrm{N}}$, arising from an interference of electromagnetic and nuclear amplitudes in the scattering matrix. Detector efficiencies and differences in beam luminosities are removed from the asymmetry ${ }^{2}$ by combining hits in the left, $N_{L}$, and right, $N_{R}$, detector hemispheres with up, $N^{\uparrow}$, and down, $N^{\downarrow}$, states of the polarization, $P$, [3]:

$$
A_{N} \cdot P=\epsilon=\frac{\sqrt{N_{L}^{\uparrow} \cdot N_{R}^{\downarrow}}-\sqrt{N_{R}^{\uparrow} \cdot N_{L}^{\downarrow}}}{\sqrt{N_{L}^{\uparrow} \cdot N_{R}^{\downarrow}}+\sqrt{N_{R}^{\uparrow} \cdot N_{L}^{\downarrow}}}
$$

The asymmetries can be used to determine the beam polarization with a known analyzing power. For symmetry under parity transformation in elastic proton-proton scattering, it is evident that $A_{N \text {,beam }}=-A_{N \text {, target }}$. With the polarization of the jet target being monitored in a separate Breit-Rabi unit, the beam polarization is then directly measurable through the respective asymmetries:

\footnotetext{
${ }^{2}$ The transverse asymmetry, $A_{N}$, leads to a cosine modulation in the azimuthal, $\varphi$, distribution in the laboratory frame. The HJET detectors are situated in transverse direction with respect to the beam polarization, $\operatorname{so} \cos \varphi \approx 1$.
} 


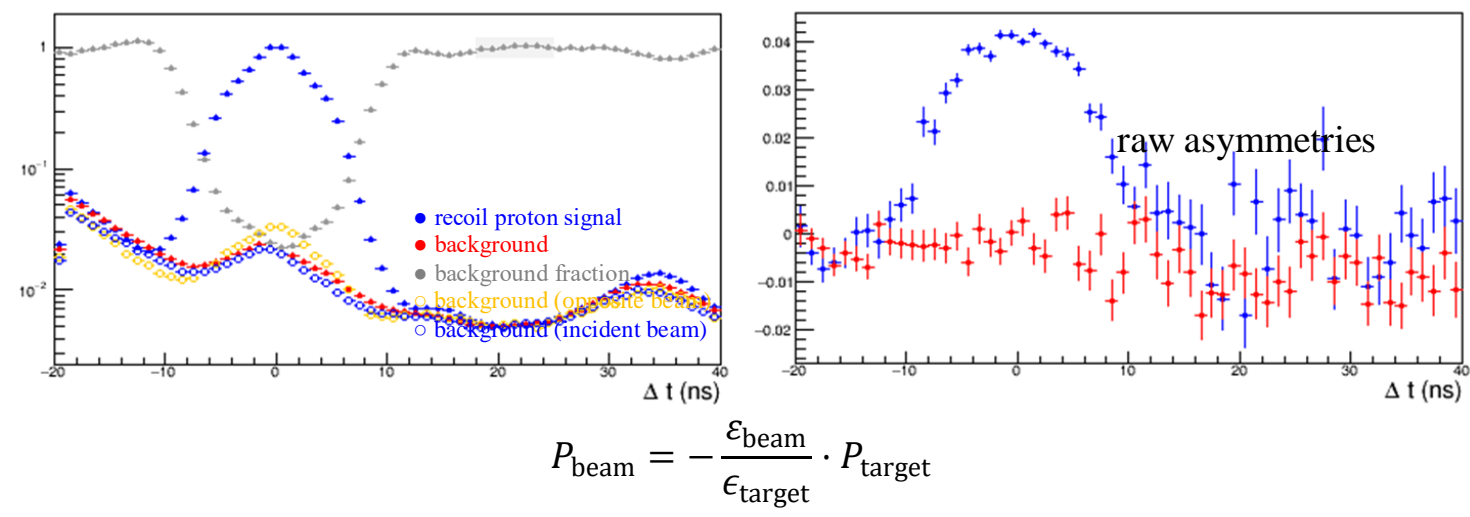

Figure 3: Examples of signal and background distributions $\left(2.0<T \_k i n<3.0 \mathrm{MeV}\right)$ as function of the difference of time of flight to a nonrelativistic proton. The background is estimated from the unfilled bunches in either beam and variations on the selection of missing mass. The background asymmetries are consistent with zero; the background fraction is used to correct for the dilution of the asymmetry of elastic proton-proton scattering.

The asymmetry is calculated as a function of the kinematic energy of the recoil proton. Events are selected with a time of flight difference of less than $5 \mathrm{~ns}$ with respect to a nonrelativistic proton of known energy. In addition, the missing mass of the event (after detector alignment) has to be within $50 \mathrm{MeV} / \mathrm{c}^{2}$ of the elastically scattered proton mass.

There are various sources of background that can potentially affect the asymmetries in different ways. As long as the background is not polarization dependent, it will not affect the direct determination of the beam polarization, though. In this case, both the beam and the target asymmetries are diluted in the same way and any polarization independent contribution cancels in the asymmetry ratio. Highly energetic particles from inelastic events are the main source of background. These particles are fast and typically leave little energy when they punch through the detector, but their spectrum can be fairly wide. There is reason to believe that this background is not polarization dependent, but previous analyses have been statistically limited. Polarization dependent background can possibly come from elastic scattering of beam gas or the far tails of the jet target. Such background would contribute differently to the target and the (two opposite) beam asymmetries.

Using the new data set from the 2015 RHIC run, these background sources can be studied in unprecedented detail. The RHIC beams potentially can consist of 120 bunches but typically only 110 of them are filled. The unfilled bunches are not aligned at the HJET polarimeter and thus can be used to estimate clean contributions from the signal beam as well as background from the opposite beam. Also, the kinematic selection can be varied to study the polarization dependence as well as the background fraction.

Figure 3 shows example distributions of the signal and background contributions as function of the time of flight (for recoil energies between 2.0 and 3.0 MeV). The elastic signal peak stands out visibly and is normalized to 1 , here, and the different background distributions are normalized relative to this between 18 and $25 \mathrm{~ns}$. The inclusive background distribution is using the same time of flight selection as the signal, but the missing mass difference is required to be larger than $120 \mathrm{MeV} / \mathrm{c}^{2}$ from the recoil proton. The same background cuts are used for the opposite beam's empty bunches, selecting only the unreducible background from the primary 
beam. The background from the opposite beam is selected with the elastic cut on the missing mass for empty bunches of the primary beam.

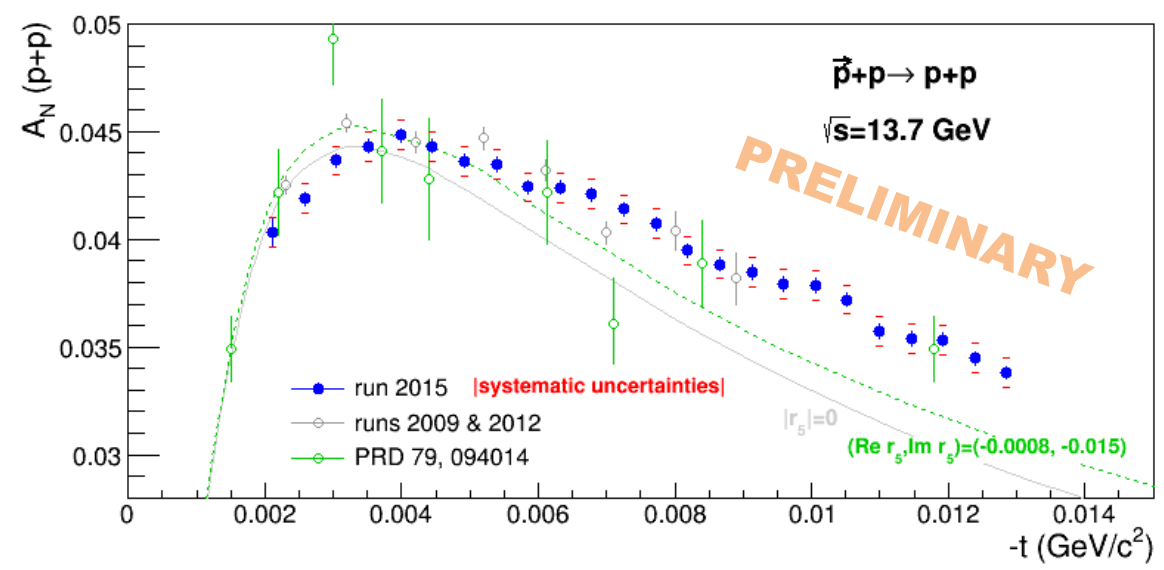

Figure 4: New results for the analyzing power in elastic proton-proton scattering as function of momentum transfer, $-t$. The new data are compared to previous published data [4] from 2004 and preliminary data from 2009/2012. The statistical uncertainties are highly improved and should enable a better determination of the amplitude $r_{5}$ in the future.

All background contributions show surprisingly similar features, namely a slight enhancement directly under the elastic peak and a smaller peak at later times. In particular they match up nicely with the signal distribution at early times which are far from the normalization and dominated by inelastic particle production. The background fractions are much improved in the current elastic event selection compared to previous analyses; they are on the order of 3-4\% during $\mathrm{p}+\mathrm{p}$ operation and slightly larger during $\mathrm{p}+\mathrm{A}$ collisions. The asymmetries are calculated for signal and background distributions. The elastic region clearly stands out with significant asymmetries on the order of a few percent. In the background, the asymmetries are overall consistent with zero. The final asymmetries are corrected for the background fraction and shown as function of momentum transfer, $-t$, in Figure 4 . The statistical precision is highly improved compared to previous results [4]. Systematic uncertainties are dominated by inconsistencies between the incident beams and different periods during the 2015 RHIC run.

The beam polarizations have been determined in a fast offline analysis during the data taking. Results in Figure 5 show preliminary beam polarizations as function of RHIC fill number during $\mathrm{p}+\mathrm{p}$ operation; the period covered ranges from early February to late April 2015. The final results with the new analysis technique will reduce the uncertainties but should be consistent with the current polarizations. 


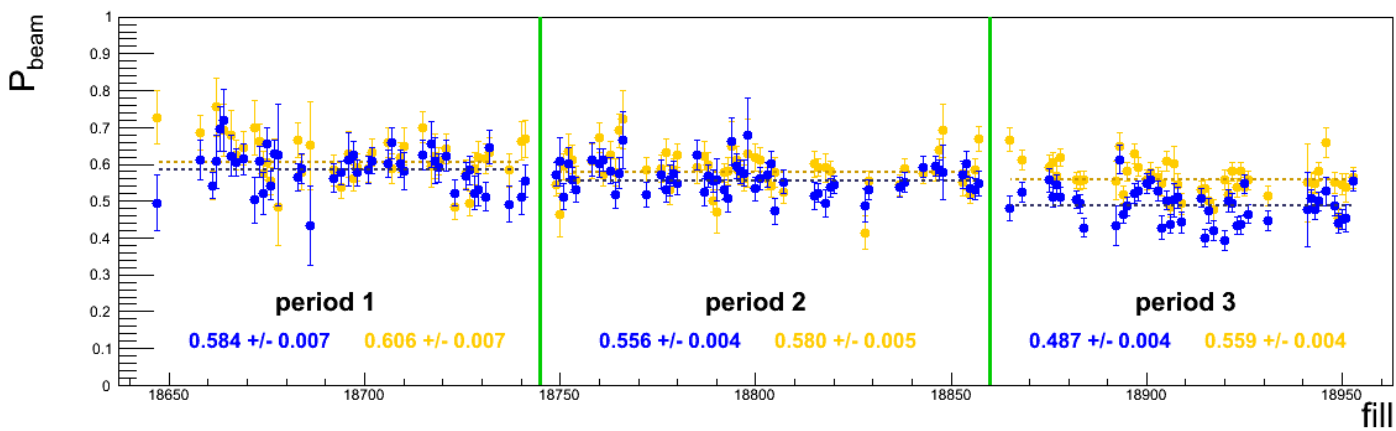

Figure 5: Preliminary beam polarizations, $P_{\text {beam }}$, from the RHIC 2015 run (as function of fill number) from the fast offline analysis. Average beam polarizations are well above 50\% over the full period. The vertial lines denote major changes in the spin rotators in the STAR experiment.

\section{Conclusions and Outlook}

The HJET detector and readout electronics have been upgraded for the recent RHIC 2015 run. This, in combination with an unprecedented recorded luminosity, enabled new systematic studies of the background contributions. The background asymmetry is generally not exhibiting any sizable polarization dependence which could affect the determination of the RHIC beam polarization severely. The background fractions are small, on the order of $3-4 \%$, and they are corrected for the extraction of the analyzing power in elastic proton-proton scattering. Compared to previous data, the new results are highly improved in statistical and systematic uncertainties. Preliminary (fast offline) results show average beam polarizations of well above 50\% for the whole run 2015 period. Differences between the two beams are understood and consistent with the accelerator parameters [5]. The final analysis of the beam polarizations will include the new cuts and analysis techniques. The main uncertainty remains the molecular component of $\mathrm{H}_{2}$ in the polarized atomic hydrogen jet target, on the order of $3 \%$, and it is desirable to monitor this part more regularly during or in between beam operations.

\section{References}

[1] G. Webb, Relative Polarization Measurements Using Thin Carbon Targets at RHIC, these proceedings.

[2] A. Poblaguev, A New DAQ System for the HJET polarimeter at RHIC, these proceedings.

[3] G.G. Ohlsen, P.W. Keaton, Techniques for Measurement of Spin-1/2 and Spin-1 Polarization Analyzing Tensores, Nucl. Instr. and Meth. 109, 41 (1973).

[4] I. Alekseev et al., Measurement of single and double spin asymmetry in elastic pp scattering in the CNI region with a polarized hydrogen gas jet target, Phys. Rev. D79, 094014 (2009).

[5] A. Zelenski, Polarized Beams in AGS and RHIC in Run-2015, these proceedings. 